\title{
The Elasticity Coefficients Measurement of Human Dentin Based on RUS
}

\author{
Fan Fan, Dandan Feng, Rui Wang, Qiang Zhang, and Haijun Niu \\ Key Laboratory of Ministry of Education for Biomechanics and Mechanobiology, School of Biological Science and Medical Engineering, \\ Beihang University, Beijing, China \\ Correspondence should be addressed to Haijun Niu; hjniu@buaa.edu.cn
}

Received 4 November 2016; Accepted 5 April 2017; Published 30 April 2017

Academic Editor: Andrea Scribante

Copyright (C) 2017 Fan Fan et al. This is an open access article distributed under the Creative Commons Attribution License, which permits unrestricted use, distribution, and reproduction in any medium, provided the original work is properly cited.

\begin{abstract}
This paper proposed to take advantages of resonant ultrasound spectroscopy (RUS) to measure the mechanical properties of human dentin specimen. The resonant spectroscopy of the dentin specimen was obtained between the frequency bands $155 \mathrm{and} 575 \mathrm{kHz}$, and resonant frequencies were extracted by linear predictive filter and then by Levenberg-Marquardt method. By inverse problem approach, 13 experimental resonant frequencies progressively matched to the first 30 orders of theoretical resonant frequencies calculated by Lagrangian variational method. The full second-order elastic tensor of dentin specimen was adjusted. The whole set of human dentin engineering moduli, including Young's moduli $\left(E_{11}=22.641 \mathrm{GPa}, E_{33}=13.637 \mathrm{GPa}\right)$, shear moduli $\left(G_{12}=\right.$ $\left.10.608 \mathrm{GPa}, G_{23}=7.742 \mathrm{Gpa}\right)$, and Poisson's ratios $\left(\nu_{12}=0.067, v_{31}=0.378\right)$, were finally calculated. This study demonstrates that RUS can be successfully adapted to measure the mechanical properties of low quality factor biomaterials.
\end{abstract}

\section{Introduction}

As the most abundant mineralized tissue in human teeth, dentin is composed of about $50 \%$ inorganic components (basically calcium hydroxyapatite), $30 \%$ organic components (mainly type I collagen fibers), and $20 \%$ water $[1,2]$. Compared with enamel, dentin has less inorganic components and thus is softer and more elastic. These characteristics ensure dentin being indispensable to cushion chewing force and protect internal pulp [3].

The researches about dentin mechanical properties began from 19th century and never stopped since then [3]. To date, the main methods of dentin mechanical properties measurement mainly included tensile and compression test $[2,4]$, acoustics method (pulse echo method, bulk wave method, elasticity imaging, etc.) [4-8], and macroscopic indentation and nanoindentation method [9-11]. These methods helped not only promote the understanding of macromechanical properties of dentin and micromechanical properties of dentin tubule, but offer important significance for the design, development, and evaluation of clinical dental restorative materials as well. Nevertheless, these methods mentioned above have their own limitations. For example, the macrotest methods required a relative larger size of the specimen, which was difficult to obtain from human dentin. Besides, although the nanoindentation method could be used to measure the elastic moduli in different directions of small-sized specimen, it mainly reflected that the mechanical properties deeply rely on the scope of indentation, which led to the differences from the macroscopic mechanical properties.

Since the 1990s, resonant ultrasound spectroscopy (RUS) has been developed as an accurate and efficient method to characterize the material properties [12-15]. The basic mechanism of RUS is to obtain a series of mechanical resonant frequencies by generating free vibrations with ultrasound excitations, then predict theoretical model frequencies with Lagrangian variational method, and finally get the material elastic properties by comparing the measured frequencies with the predicted ones (inverse problem approach). RUS has been regarded as the gold standard for measuring the elastic moduli of high $Q$ (quality factor) solid materials. And it beats other methods by the following three advantages: (1) The full elastic tensor could be assessed from a single sample in a single nondestructive experiment over other elasticity measurement methods; (2) RUS was well-adapted to small-sized samples (a few millimeters or less); (3) The measurement results 
were more repeatable and accurate $[12,14,16]$. In recent years, Pascal's group were dedicated to measuring the elastic coefficients of cortical bone by RUS, which made it possible for breaking the limitations when measuring low-Q materials by RUS $[12,13,17]$.

In this paper, the method of human dentin elasticity extraction based on RUS was studied. Firstly, the resonant spectroscopy of dentin specimen was obtained by ultrasound experiment. Then, the resonant frequencies were extracted through signal processing of linear predictive filter and then by nonlinear least squares method (Levenberg-Marquardt method). Combined with the theoretical resonant frequencies calculated by Lagrangian variational method, inverse problem approach was introduced to obtain the complete second-order elastic tensor of dentin samples. The engineering moduli, including Young's moduli, shear moduli, and Poisson's ratios, were finally calculated.

\section{Materials and Methods}

2.1. Specimen Preparations. The tooth used in this paper was complete, fresh, and caries-free. It was a left upper third molar from a 25-year-old male, which was collected from Beihang Hospital. The donor provided consent to donate his tooth for this study. The entire experimental procedures were approved by the Institutional Animal Care and Use Committee of Beihang University and performed under the guidelines of the National Institutes of Health.

Before experimentation began, the specimen was stored in saline solution at room temperature $\left(22^{\circ} \mathrm{C}\right)$. After cleaning the entire tooth surface by removing calculus and granulation, the dentin part was cut into a rectangular parallelepiped by a low speed diamond cutting machine (SYJ-150, Shenyang Kejing Auto-Instrument Co., Ltd., Shenyang, China) with $0.01 \mathrm{~mm}$ positioning accuracy and $25-300 \mathrm{rad} / \mathrm{min}$ rotational speed and a whole sintered diamond saw blade $(0.3 \mathrm{~mm}$ thick). The six faces of the specimen were polished with P500, P800, and P1000 abrasive paper progressively. With repeated protractor measurements of each corner of the specimen and polish, a standard rectangular parallelepiped human dentin specimen was obtained (accuracy: 90 $\pm 0.5^{\circ}$ ) [12]. The mass of the specimen was $111 \mathrm{mg}$, and the dimensions were $5.696 \mathrm{~mm} \times 3.620 \mathrm{~mm} \times 2.704 \mathrm{~mm}$.

2.2. Theoretical Resonant Frequencies Calculation. The resonant frequencies of solid material are related to many factors such as density, dimensions, elastic tensor, and boundary conditions. Moreover, the relationship among these factors is nonlinear and no analytical solutions exist either. To figure out the approximate numerical solutions, Lagrange variational method was imported here. As (1) shows, the resonant angular frequencies $\omega$ could be calculated by searching for the stationary points of the Lagrangian $L$ under free-surface boundary condition $[14,18-21]$.

$$
L=\int\left(E_{k}-E_{p}\right) d V
$$

where $E_{k}$ and $E_{p}$ are kinetic energy and potential energy, respectively, given by

$$
\begin{aligned}
& E_{k}=\frac{1}{2} \sum_{i} \rho \omega^{2} u_{i}^{2}, \\
& E_{p}=\frac{1}{2} \sum_{i, j, k, l} c_{i j k l} \frac{\partial u_{i}}{\partial x_{j}} \frac{\partial u_{k}}{\partial x_{l}} .
\end{aligned}
$$

In (2), $\rho$ and $V$ are the specimen's density and volume, respectively, $u_{i}$ is the component of the displacement field in Cartesian coordinates, and $c_{i j k l}$ are the stiffness constants of solid material.

Kinney et al. found the elastic constants $C_{i j}$ of hydrated dentin exhibited as transverse isotropy, with five independent constants: $C_{11}, C_{12}, C_{13}, C_{33}$, and $C_{44}$, as (3) shows $[1,22]$ :

$$
C_{i j}=\left(\begin{array}{cccccc}
C_{11} & C_{12} & C_{13} & 0 & 0 & 0 \\
C_{12} & C_{11} & C_{13} & 0 & 0 & 0 \\
C_{13} & C_{13} & C_{33} & 0 & 0 & 0 \\
0 & 0 & 0 & C_{44} & 0 & 0 \\
0 & 0 & 0 & 0 & C_{44} & 0 \\
0 & 0 & 0 & 0 & 0 & \frac{1}{2}\left(C_{11}-C_{12}\right)
\end{array}\right) .
$$

To find the stationary point of the Lagrangian $L$, (4) was calculated:

$$
\delta L=0 .
$$

To solve the numerical solutions of (4), by expanding the displacement field $u_{i}$ to a set of polynomial functions, Rayleigh-Ritz method was introduced:

$$
u_{i}=\sum_{\lambda} a_{i \lambda} \phi_{\lambda}
$$

where the choice of $\phi_{\lambda}$ is rather arbitrary.

Historically, Visscher et al. found that there was none simpler than powers of the Cartesian coordinates when expanding the displacement field [18], so a set of power exponent functions were chosen for simplifying computing:

$$
\phi_{\lambda}=x^{l} y^{m} z^{n}
$$

where $l+m+n \leq N$.

When $N \rightarrow+\infty$, the solutions of (4) are the exact solutions. Considering a good compromise between computational accuracy and computing time, $N$ was chosen as 12 .

Based on (5) and (6), (4) was transferred to the generalized eigenvalue problem:

$$
\omega^{2} E a=\Gamma a,
$$

where $E$ and $\Gamma$ are expressed as follows:

$$
\begin{aligned}
& E_{\lambda i \lambda^{\prime} i^{\prime}}=\delta_{i i^{\prime}} \rho \int_{V} x^{l+l^{\prime}} y^{m+m^{\prime}} z^{n+n^{\prime}} d V \\
& \Gamma_{\lambda i \lambda^{\prime} i^{\prime}}=\sum_{j, j^{\prime}} C_{i j i^{\prime} j^{\prime}} \int_{V} \frac{\partial x^{l} y^{m} z^{n}}{\partial x_{j}} \frac{\partial x^{l^{\prime}} y^{m^{\prime}} z^{n^{\prime}}}{\partial x_{j^{\prime}}} d V .
\end{aligned}
$$




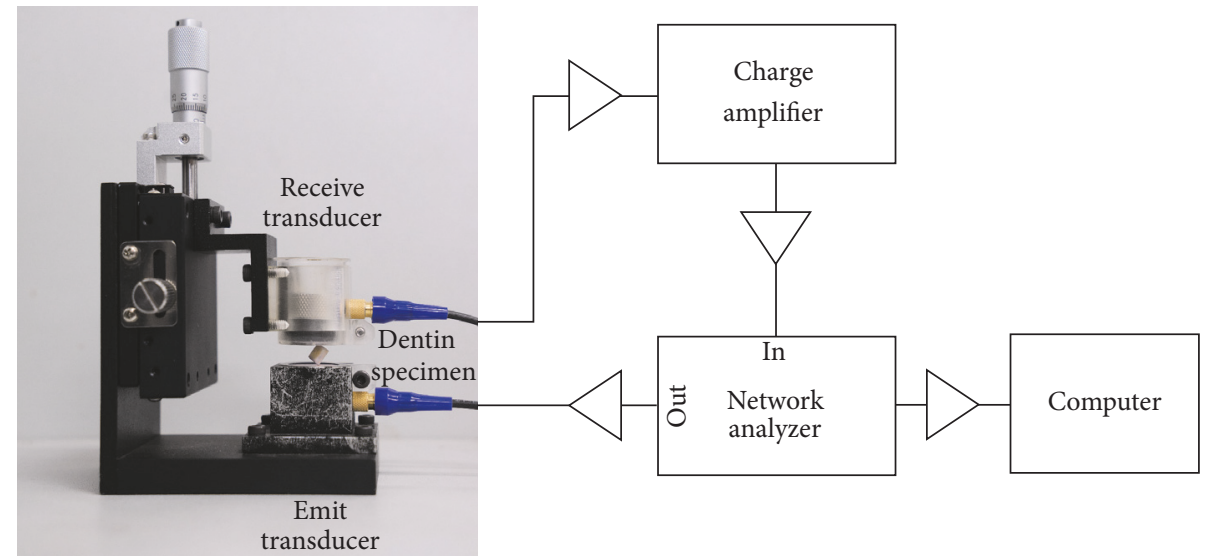

FIGURE 1: RUS experiment platform: photograph of the dentin specimen mounted between emitting and receiving transducers and block diagram of other hardware.

In the end, the theoretical values of solid resonant frequency could be calculated by solving (7).

Before the resonance experiment, a set of initial elastic tensors combined with the density and dentin specimen dimensions were needed to be set; then the theoretical resonant frequencies could be calculated later. The initial elastic tensor set plays a decisive role in iterative efficiency and accuracy, so it is indispensable to find an exact-value-closest set as shown in matrix (9) $[18,22]$. In this paper, the first 30 frequencies' range was chosen as the experimental frequency sweep range reference.

$$
\left(\begin{array}{cccccc}
42.6 & 25.4 & 19.7 & 0 & 0 & 0 \\
25.4 & 42.6 & 19.7 & 0 & 0 & 0 \\
19.7 & 19.7 & 34.6 & 0 & 0 & 0 \\
0 & 0 & 0 & 9.4 & 0 & 0 \\
0 & 0 & 0 & 0 & 9.4 & 0 \\
0 & 0 & 0 & 0 & 0 & 8.6
\end{array}\right) .
$$

2.3. RUS Experiment. The RUS experiment platform is shown in Figure 1. The platform was made just to fit the free-surface boundary condition. The dentin specimen was mounted on opposing corners between two shear wave contact transducers (V154RM, Panametrics Inc., Waltham, US) in the RUS system. A network analyzer (Bode 100, Omicron electronics $\mathrm{GmbH}$, Klaus, Austria) was used to output a swept-frequency signal between $155 \mathrm{kHz}$ and $575 \mathrm{kHz}$ (frequency resolution: $30 \mathrm{~Hz}$ ) as the excitation of the transmit transducer. The frequency response of the specimen was received by the other transducer, amplified by a broadband charge amplifier (HQA15M-10T, Femto Messtechnik GmbH, Berlin, Germany), sent back to the network analyzer, and recorded [12].
2.4. Experimental Resonant Frequencies Extraction. The frequency response FR acquired by the network analyzer was modeled as a sum of $M$ Lorentzian lineshapes [12]:

$$
\mathrm{FR}(f)=\sum_{k=1}^{M} \frac{a_{k}}{\left(f_{k}^{2}-f^{2}\right)+i\left(f_{k} f / Q_{k}\right)}
$$

where $a_{k}$ are the complex amplitudes, $f_{k}$ are the resonant frequencies, and $Q_{k}$ are the resonant quality factors.

When RUS was used on low damping (high Q) materials, the resonant frequencies $f_{k}$, the sharp peak, could be easily recognized from the resonant spectrum. For those low $Q$ materials such as dentin, peaks may be broad and overlap each other, which made it difficult to directly extract resonant frequencies from spectrum. Therefore linear predictive filter, an accurate signal processing method, introduced by Kumaresan, Tufts, and Lebedev et al. was selected here to distinguish the resonant frequencies, which was proved to be a perfect solution [23-27].

The frequency response FR was converted to $y(n)$ in time domain by $N$-point inverse Fourier transform $(N$ is the length of the FR data), and then matrix $A$ was created based on

$$
A=\left(\begin{array}{cccc}
y(L) & y(L-1) & \cdots & y(1) \\
y(L+1) & y(L) & \cdots & y(2) \\
\vdots & \vdots & \ddots & \vdots \\
y(N-1) & y(N-2) & \cdots & y(N-L)
\end{array}\right) .
$$

According to the linear prediction method, the first $L$ points of $y(n)$ contained enough information to predict values of the others. So the linear predictive filter equation could be set as

$$
A g=b,
$$


where $b$ has the definition:

$$
b=\left(\begin{array}{c}
y(L+1) \\
y(L+2) \\
\vdots \\
y(N)
\end{array}\right)
$$

and $g$ is a column vector with $L$ components:

$$
g=\left(\begin{array}{c}
g_{1} \\
g_{2} \\
\vdots \\
g_{L}
\end{array}\right) .
$$

Then the transfer function of this linear predictive filter could be written as

$$
H(z)=1+\sum_{k=1}^{L} g_{k} z^{-k}
$$

The predicted values of the resonant frequency $f_{k}$ and the quality factor $Q_{k}$ could be obtained by finding the zeros outside the unit circle in $Z$ domain. Substituting the predicted $f_{k}$ and $Q_{k}$ into (10), complex resonant amplitude $a_{k}$ and also the predicted frequency spectrum FRlp could be calculated. One of the advantages of this method was that there was no need to know the exact numbers of resonant peaks in FR, but some differences existed between FRlp and FR. To figure out these differences, a Levenberg-Marquardt method was taken to optimize the parameters, and we considered the $f_{k}$ 's which minimized (16), the true resonant frequencies [28].

$$
F(f)=\sum\left(\frac{\mathrm{FR} l p(f)-\mathrm{FR}(f)}{\mathrm{FR}(f)}\right)^{2} .
$$

During a single experiment, some resonant mode might not be excited, so 7 measurements on the dentin specimen, by remounting it on opposing corners each time, were conducted for a good reproducibility. Each of the regarded experimental resonant frequencies $f^{\text {exp }}$ was present in at least two frequency responses in the 7 measurements.

2.5. Elastic Tensor Calculation. Levenberg-Marquardt nonlinear optimization inverse problem approach was selected for the purpose of calculating the elastic tensor [14, 28, 29]. Here the cost function was introduced as a criterion for the iteration, and the value of the cost function was calculated, as shown in

$$
F(C)=\sum_{i=1}^{N} w_{i}\left(f_{i}^{\exp }-f_{i}^{\mathrm{cal}}(C)\right)^{2}
$$

where $C$ is an independent component of the elastic tensor, $N$ is the number of the resonant frequency, $f_{i}^{\text {cal }}$ is the $i$ th order calculated resonance frequency, $f_{i}^{\exp }$ is the $i$ th order experimental resonance frequency, and $w_{i}$ is weighting factor, expressed as follows:

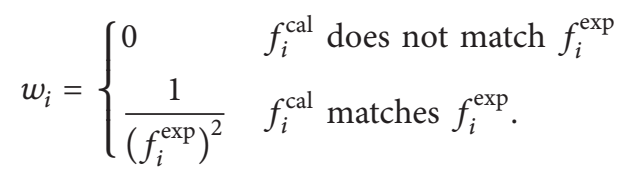

The iterative process was completed when the experimental and theoretical frequencies matched perfectly. In other words, cost function reached the global minimum and also became convergent.

2.6. Engineering Moduli Calculation. The $6 \times 6$ stiffness matrix was constructed and numerically inverted to obtain the compliance matrix $C_{i j}^{-1}$, from which engineering moduli could be calculated.

$$
C_{i j}^{-1}=\left(\begin{array}{cccccc}
\frac{1}{E_{11}} & \frac{-v_{12}}{E_{11}} & \frac{-v_{31}}{E_{33}} & 0 & 0 & 0 \\
\frac{-v_{12}}{E_{11}} & \frac{1}{E_{11}} & \frac{-v_{31}}{E_{33}} & 0 & 0 & 0 \\
\frac{-v_{31}}{E_{33}} & \frac{-v_{31}}{E_{33}} & \frac{1}{E_{33}} & 0 & 0 & 0 \\
0 & 0 & 0 & \frac{1}{G_{23}} & 0 & 0 \\
0 & 0 & 0 & 0 & \frac{1}{G_{23}} & 0 \\
0 & 0 & 0 & 0 & 0 & \frac{1}{G_{12}}
\end{array}\right),
$$

where $E_{i i}$ 's are Young's moduli (GPa), $G_{i j}$ 's are shear moduli $(\mathrm{GPa})$, and $v_{i j}$ 's are Poisson's ratios.

\section{Result and Discussion}

3.1. Results of Experimental Resonant Frequencies Extractions. In accordance with the method of 2.4, in each of 7 measurements, two or more resonant frequencies with similar $Q$ values were selected, and their mean values and standard deviations were calculated from 2 to 7 values depending on the frequency. As shown in Table 1, when the standard deviation (column 2) was less than $0.5 \%$, the mean value (column 1) was retained as the resonant frequencies extracted from the experiment.

Figure 2 shows the measured frequency responses of the dentin specimen between 155 and $575 \mathrm{kHz}$. The 16 calculated resonant frequencies, presented at least two times, are represented as $*$. The mean values are represented as the vertical line.

3.2. Results of the Inverse Problem. After nonlinear optimization, the theoretical values of resonant frequency were obtained and shown in column 4 of Table 2. Among the first 30 orders of the calculated resonant frequencies, 13 can be paired with the measured frequencies. The root-mean-square error between calculated and experimental frequencies was below $0.65 \%$. 


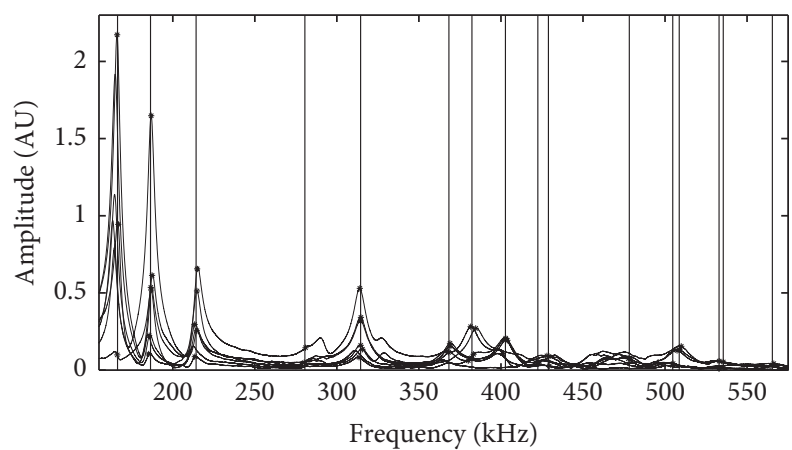

FIgURE 2: Measured frequency response of the dentin specimen between 155 and $575 \mathrm{kHz}$ and the 16 resonant frequencies distributions. $*$ : the calculated resonant frequencies presented at least two times. Vertical line: the mean values of the resonant frequencies.

TABLE 1: Results of 16 experimental resonant frequencies' extraction and $Q$ factors.

\begin{tabular}{lcc}
\hline$f^{\text {exp }} / \mathrm{kHz}$ & SD $\%$ & $Q$ \\
\hline 166.34 & 0.22 & 45.56 \\
186.44 & 0.43 & 46.05 \\
214.20 & 0.36 & 45.40 \\
280.50 & 0.20 & 47.98 \\
314.47 & 0.22 & 42.05 \\
368.28 & 0.23 & 46.41 \\
382.30 & 0.45 & 44.57 \\
402.73 & 0.29 & 48.32 \\
422.47 & 0.17 & 46.21 \\
428.92 & 0.19 & 40.78 \\
478.22 & 0.18 & 44.81 \\
504.72 & 0.06 & 42.28 \\
508.66 & 0.25 & 45.63 \\
532.94 & 0.01 & 53.49 \\
535.48 & 0.02 & 39.95 \\
565.41 & 0.22 & 48.76 \\
\hline
\end{tabular}

The correspondence elastic tensor is shown in matrix (20), with the unit of GPa.

$$
\left(\begin{array}{cccccc}
35.27 & 14.06 & 18.63 & 0 & 0 & 0 \\
14.06 & 35.27 & 18.63 & 0 & 0 & 0 \\
18.63 & 18.63 & 27.71 & 0 & 0 & 0 \\
0 & 0 & 0 & 7.74 & 0 & 0 \\
0 & 0 & 0 & 0 & 7.74 & 0 \\
0 & 0 & 0 & 0 & 0 & 10.61
\end{array}\right) .
$$

3.3. Results of Engineering Moduli Calculations. In accordance with (19), engineering moduli could be calculated as shown in Table 3. Column 3 also listed the engineering moduli results of Kinney et al.s work [22].

3.4. Discussion. In this paper, the method of human dentin elasticity extraction based on RUS was studied. Firstly, the
TABLE 2: Results of the original calculated, experimental, and optimized calculated resonant frequencies.

\begin{tabular}{|c|c|c|c|c|}
\hline Mode & Original $f^{\mathrm{cal}} / \mathrm{kHz}$ & $f^{\exp } / \mathrm{kHz}$ & Final $f^{\mathrm{cal}} / \mathrm{kHz}$ & Err\% \\
\hline 1 & 161.13 & 166.34 & 165.53 & -0.48 \\
\hline 2 & 195.31 & 186.44 & 186.62 & 0.10 \\
\hline 3 & 220.66 & 214.20 & 213.07 & -0.53 \\
\hline 4 & 292.13 & 280.50 & 281.66 & 0.42 \\
\hline 5 & 294.42 & - & 312.63 & - \\
\hline 6 & 313.36 & 314.47 & 313.06 & -0.45 \\
\hline 7 & 333.39 & - & 315.26 & - \\
\hline 8 & 366.55 & - & 342.12 & 一 \\
\hline 9 & 385.12 & - & 350.58 & - \\
\hline 10 & 412.00 & 368.28 & 368.17 & -0.03 \\
\hline 11 & 414.05 & - & 370.08 & - \\
\hline 12 & 416.61 & 382.30 & 384.59 & 0.60 \\
\hline 13 & 418.61 & - & 386.37 & - \\
\hline 14 & 438.89 & 402.73 & 401.68 & 0.08 \\
\hline 15 & 445.36 & - & 403.31 & - \\
\hline 16 & 457.02 & 422.47 & 422.80 & 0.08 \\
\hline 17 & 457.20 & 428.92 & 428.06 & -0.20 \\
\hline 18 & 484.41 & - & 430.71 & - \\
\hline 19 & 484.84 & - & 451.03 & - \\
\hline 20 & 496.81 & - & 453.71 & - \\
\hline 21 & 498.90 & - & 453.81 & - \\
\hline 22 & 516.59 & - & 476.06 & - \\
\hline 23 & 528.65 & 478.22 & 477.13 & -0.23 \\
\hline 24 & 551.61 & - & 485.94 & - \\
\hline 25 & 557.68 & - & 490.54 & - \\
\hline 26 & 560.68 & - & 495.24 & - \\
\hline 27 & 563.08 & 504.72 & 506.36 & 0.33 \\
\hline 28 & 565.01 & 508.66 & 511.92 & 0.64 \\
\hline 29 & 568.89 & - & 521.58 & - \\
\hline 30 & 569.86 & - & 531.23 & - \\
\hline
\end{tabular}

TABLE 3: Results of engineering moduli calculations.

\begin{tabular}{lcc}
\hline & Modulus & Kinney et al. [22] \\
\hline$E_{11} / \mathrm{GPa}$ & 22.641 & 25 \\
$E_{33} / \mathrm{GPa}$ & 13.637 & 23.2 \\
$G_{12} / \mathrm{GPa}$ & 10.608 & 8.6 \\
$G_{23} / \mathrm{GPa}$ & 7.742 & 9.4 \\
$\nu_{12}$ & 0.067 & 0.45 \\
$\nu_{31}$ & 0.378 & 0.29 \\
\hline
\end{tabular}

resonant spectroscopy between 155 and $575 \mathrm{kHz}$ of a dentin specimen was obtained. The resonant frequencies were extracted by linear predictive filter and then by nonlinear least squares method (Levenberg-Marquardt method). Combined with the theoretical resonant frequencies calculated by Lagrangian variational method, inverse problem approach was introduced to obtain the complete second-order elastic tensor of the dentin sample. Young's moduli, shear moduli, and Poisson's ratios were finally calculated. 
In theory, the lower order resonant modes are mostly shear modes, so, in the experiment, the shear wave ultrasound transducer could get stronger signal and more accurate frequency response [30]. As shown in Figure 2, the first three orders of resonant frequencies are more obvious and the resonance peaks are sharper, but the subsequent resonance peaks become broad due to the low quality factor of dentin. When the experimental resonant frequency was extracted, the linearly predictive filtering and nonlinear least squares optimization of the experimental frequency response could make it possible for the originally gentle and overlapping resonance peaks to be distinguished.

In general, when choosing the resonant order to be calculated, the number should be at least five times the number of unknown independent elastic constants $[12,14]$. For transversely isotropic solids with five independent constants, it is reasonable to choose the theoretical value of the first 30-order resonant frequency in the calculations. During the Levenberg-Marquardt iteration, the elastic tensor changes constantly, leading to the the first 30-order calculated frequency band being smaller, in which only 13 orders could match with the first 13 out of the whole 16 measured resonant frequencies. In this paper, we compared each experimental resonance frequency with the theoretical resonance frequencies and tried to figure out all the pairings. But interestingly, in most cases, the cost function will not converge to the minimum value if the pairing is incorrect. Using this method, the error of each matched pairing between experimental resonance frequency and calculated resonance frequency was all less than $0.65 \%$, which is in accordance with the $0.8 \%$ criterion described by Migliori and Maynard [30].

Comparing the result of this study, matrix (20), with the result of Kinney et al.s group [22], shown as matrix (9), the original elastic tensor, there are differences between each independent elastic constant. We guessed that one reason is that the experimental resonant frequency extractions after the signal processing might be more accurate, which lead to the difference compared to the extraction without signal processing method of Kinney et al's work. Another reason might be the individual differences among different teeth. The differences in elastic tensors also resulted in some differences in engineering moduli, mainly on $E_{33}$ and $\nu_{12}$.

Bernard et al. added probabilistic methods in the pairing process $[13,31]$, in which simulated annealing algorithm was introduced to compute the possibility of the pairing and then the calculated value could be automatically matched to the experimental value according to the probability. In our future work, we will try to introduce this algorithm into pairing process and explore the differences and advantages of present pairing methods. Moreover, we will also try to use more specimens to learn structure-function relationships and find other verification methods to RUS.

\section{Conclusion}

In conclusion, the elastic tensor, even Young's moduli, shear moduli, and Poisson's ratios of dentin specimen, can be accurately extracted by the signal processing method and inverse problem approach, which demonstrates that RUS is suitable for the mechanical properties measurement of low quality factor biomaterials and can provide a theoretical basis for the development of clinical dental restorative materials and the design of dental prostheses.

\section{Conflicts of Interest}

The authors have no conflicts of interest related to the manuscript.

\section{Acknowledgments}

This work was supported by the National Natural Science Foundation of China (31570945) and National Science and Technology Ministry of Science and Technology Support Program (2015BAI06B02).

\section{References}

[1] J. H. Kinney, S. J. Marshall, and G. W. Marshall, "The mechanical properties of human dentin: a critical review and re-evaluation of the dental literature," Critical Reviews in Oral Biology \& Medicine, vol. 14, no. 1, pp. 13-29, 2003.

[2] D. Zaytsev, A. S. Ivashov, J. V. Mandra, and P. Panfilov, "On the deformation behavior of human dentin under compression and bending," Materials Science \& Engineering C Materials for Biological Applications, vol. 41, pp. 83-90, 2014.

[3] Y.-R. Zhang, W. Du, X.-D. Zhou, and H.-Y. Yu, "Review of research on the mechanical properties of the human tooth," International Journal of Oral Science, vol. 6, no. 2, pp. 61-69, 2014.

[4] D. Zaytsev and P. Panfilov, "Deformation behavior of human dentin in liquid nitrogen: a diametral compression test," Materials Science \& Engineering C Materials for Biological Applications, vol. 42, pp. 48-51, 2014.

[5] T. Lee, R. Lakes S, and A. Lal, "Investigation of bovine bone by resonant ultrasound spectroscopy and transmission ultrasound," Biomechanics \& Modeling in Mechanobiology, vol. 1, no. 2, pp. 165-175, 2002.

[6] T. Watanabe, M. Miyazaki, H. Inage, and H. Kurokawa, "Determination of elastic modulus of the components at dentin-resin interface using the ultrasonic device," Dental Materials Journal, vol. 23, no. 3, pp. 361-367, 2004.

[7] M.-H. Lu, Y.-P. Zheng, and Q.-H. Huang, "A novel method to obtain modulus image of soft tissues using ultrasound water jet indentation: a phantom study," IEEE Transactions on Biomedical Engineering, vol. 54, no. 1, pp. 114-121, 2007.

[8] J. Du, X. L. Mao, P. F. Ye et al., "Three-dimensional reconstruction and visualization of human enamel ex vivo using highfrequency ultrasound," Journal of Medical \& Biological Engineering, vol. 37, no. 1, pp. 112-122, 2017.

[9] D. S. Brauer, J. F. Hilton, G. W. Marshall, and S. J. Marshall, "Nano-and micromechanical properties of dentine: investigation of differences with tooth side," Journal of Biomechanics, vol. 44, no. 8, pp. 1626-1629, 2011.

[10] T. Inoue, M. Saito, M. Yamamoto et al., "Comparison of nanohardness between coronal and radicular intertubular dentin," Dental Materials Journal, vol. 28, no. 3, pp. 295-300, 2009.

[11] L. Angker, M. V. Swain, and N. Kilpatrick, "Micro-mechanical characterisation of the properties of primary tooth dentine," Journal of Dentistry, vol. 31, no. 4, pp. 261-267, 2003. 
[12] S. Bernard, Q. Grimal, and P. Laugier, "Accurate measurement of cortical bone elasticity tensor with resonant ultrasound spectroscopy," Journal of the Mechanical Behavior of Biomedical Materials, vol. 18, pp. 12-19, 2013.

[13] S. Bernard, Q. Grimal, and P. Laugier, "Resonant ultrasound spectroscopy for viscoelastic characterization of anisotropic attenuative solid materials," Journal of the Acoustical Society of America, vol. 135, no. 5, pp. 2601-2613, 2014.

[14] A. Migliori and J. L. Sarrao, Resonant Ultrasound Spectroscopy: Applications to Physics, Materials Measurements, and Nondestructive Evaluation, Wiley, New York, NY, USA, 1997.

[15] A. Migliori, J. L. Sarrao, W. M. Visscher et al., "Resonant ultrasound spectroscopic techniques for measurement of the elastic moduli of solids," Physica B: Physics of Condensed Matter, vol. 183, no. 1-2, pp. 1-24, 1993.

[16] P. Sedlák, H. Seiner, J. Zídek, M. Janovská, and M. Landa, "Determination of all 21 independent elastic coefficients of generally anisotropic solids by resonant ultrasound spectroscopy: benchmark examples," Experimental Mechanics, vol. 54, no. 6, pp. 1073-1085, 2014.

[17] S. Bernard, Q. Grimal, and P. Laugier, "Development and validation of resonant ultrasound spectroscopy for the measurement of cortical bone elasticity on small cylindrical samples," Journal of the Acoustical Society of America, vol. 133, no. 5, pp. 35853593, 2013.

[18] W. M. Visscher, A. Mialiori, T. M. Bell, and R. A. Reinert, "On the normal modes of free vibration of inhomogeneous and anisotropic elastic objects," Journal of the Acoustical Society of America, vol. 90, no. 4, pp. 2154-2162, 1991.

[19] H. H. Demarest, "Cube-resonance method to determine the elastic constants of solids," Journal of the Acoustical Society of America, vol. 49, no. 3B, pp. 768-775, 1971.

[20] R. Holland, "Resonant properties of piezoelectric ceramic rectangular parallelepipeds," Journal of the Acoustical Society of America, vol. 43, no. 5, pp. 988-997, 1968.

[21] I. Ohno, "Free vibration of a rectangular parallelepiped crystal and its application to determination of elastic constants of orthorhombic crystals," Journal of Physics of the Earth, vol. 24, no. 4, pp. 355-379, 1976.

[22] J. H. Kinney, J. R. Gladden, G. W. Marshall, S. J. Marshall, J. H. So, and J. D. Maynard, "Resonant ultrasound spectroscopy measurements of the elastic constants of human dentin," Journal of Biomechanics, vol. 37, no. 4, pp. 437-441, 2004.

[23] A. V. Lebedev, L. A. Ostrovskiǔ, A. M. Sutin, I. A. Soustova, and P. A. Johnson, "Resonant acoustic spectroscopy at low Q factors," Acoustical Physics, vol. 49, no. 1, pp. 81-87, 2003.

[24] R. Kumaresan and D. W. Tufts, "Estimating the parameters of exponentially damped sinusoids and pole-zero modeling in noise," IEEE Transactions on Acoustics, Speech, and Signal Processing, vol. 30, no. 6, pp. 833-840, 1982.

[25] R. Kumaresan, "On the zeros of the linear prediction-error filter for deterministic signals," IEEE Transactions on Acoustics Speech \& Signal Processing, vol. 31, no. 1, pp. 217-220, 1983.

[26] A. V. Lebedev, "Method of linear prediction in the ultrasonic spectroscopy of rock," Acoustical Physics, vol. 48, no. 3, pp. 339$346,2002$.

[27] L. Ostrovsky, A. Lebedev, A. Matveyev et al., "Application of three-dimensional resonant acoustic spectroscopy method to rock and building materials," Journal of the Acoustical Society of America, vol. 110, no. 4, pp. 1770-1777, 2001.
[28] K. Madsen, H. B. Nielsen, and O. Tinglesff, Methods for NonLinear Least Squares Problems, Informatics and Mathematical Modelling, Technical University of Denmark, Lyngby, Denmark, 2004.

[29] A. Tarantola, Inverse Problem Theory and Methods for Model Parameter Estimation, SIAM: Society for Industrial and Applied Mathematics, Philadelphia, PA, USA, 2004.

[30] A. Migliori and J. D. Maynard, "Implementation of a modern resonant ultrasound spectroscopy system for the measurement of the elastic moduli of small solid specimens," Review of Scientific Instruments, vol. 76, no. 12, pp. 1-8, 2005.

[31] S. Bernard, G. Marrelec, P. Laugier, and Q. Grimal, "Bayesian normal modes identification and estimation of elastic coefficients in resonant ultrasound spectroscopy," Inverse Problems, vol. 31, no. 6, pp. 805-815, 2015. 

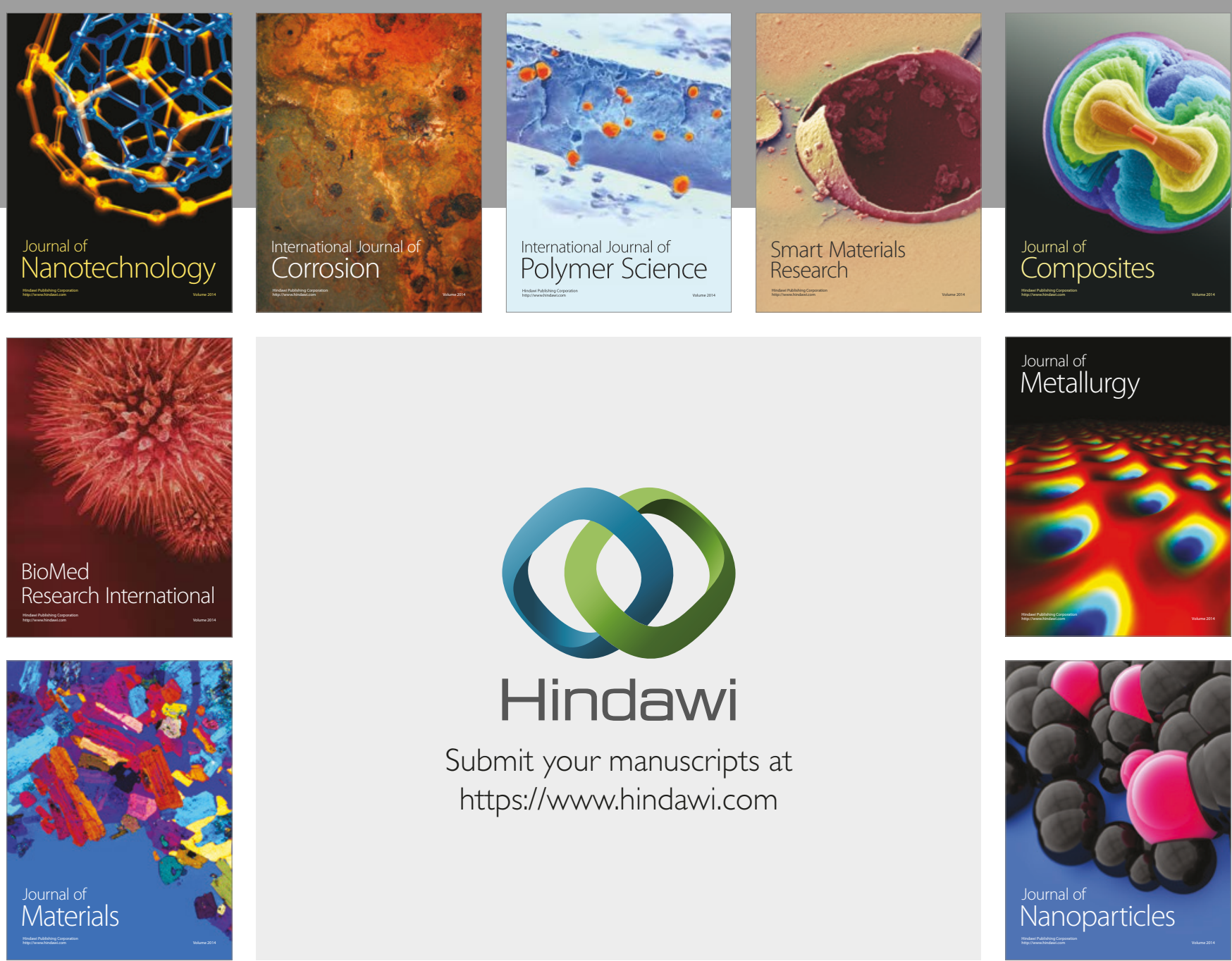

\section{Hindawi}

Submit your manuscripts at

https://www.hindawi.com
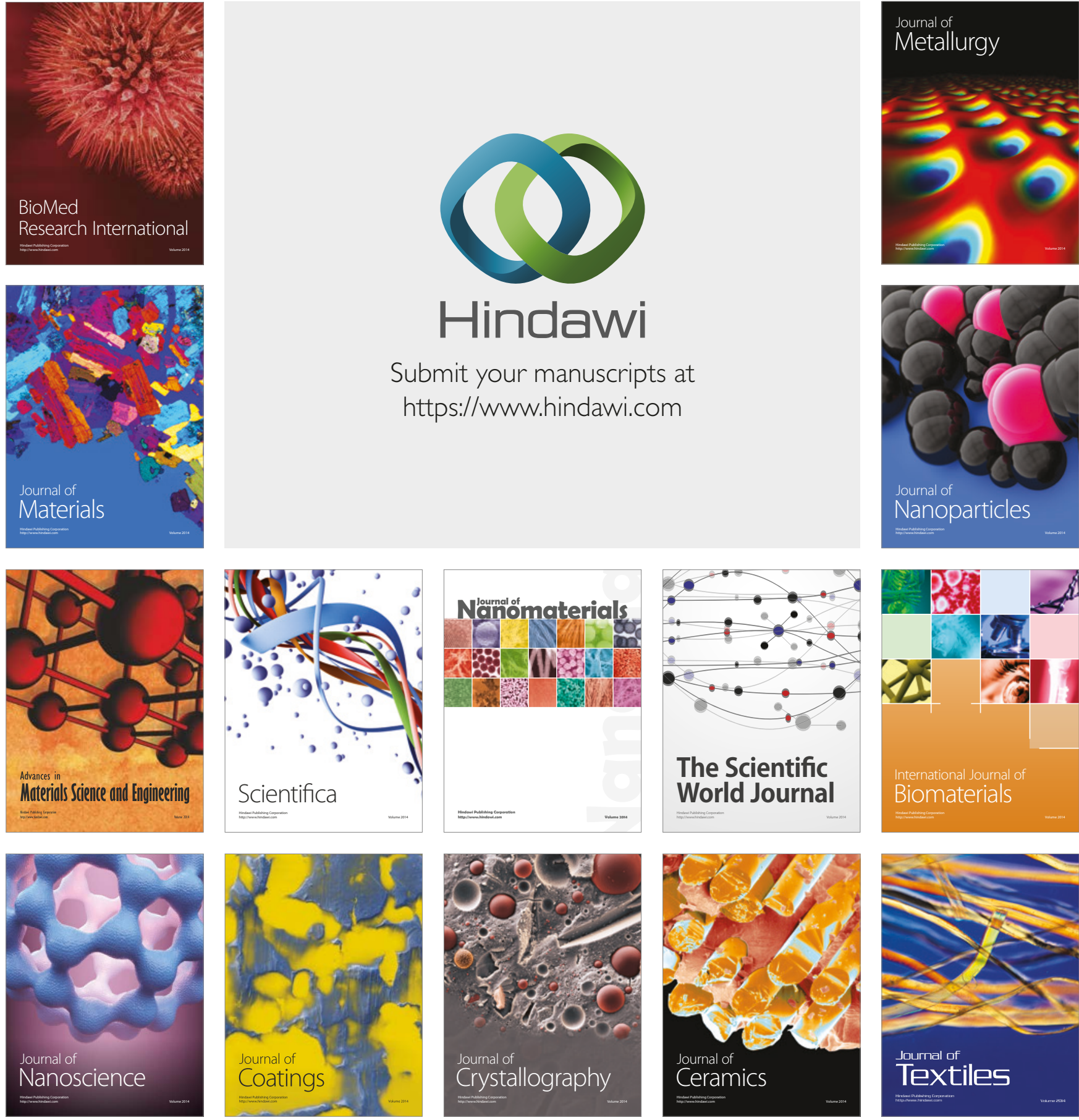

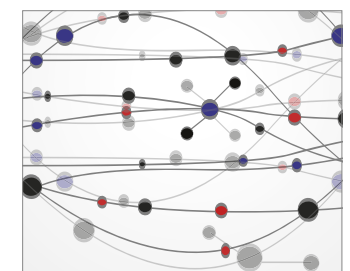

The Scientific World Journal
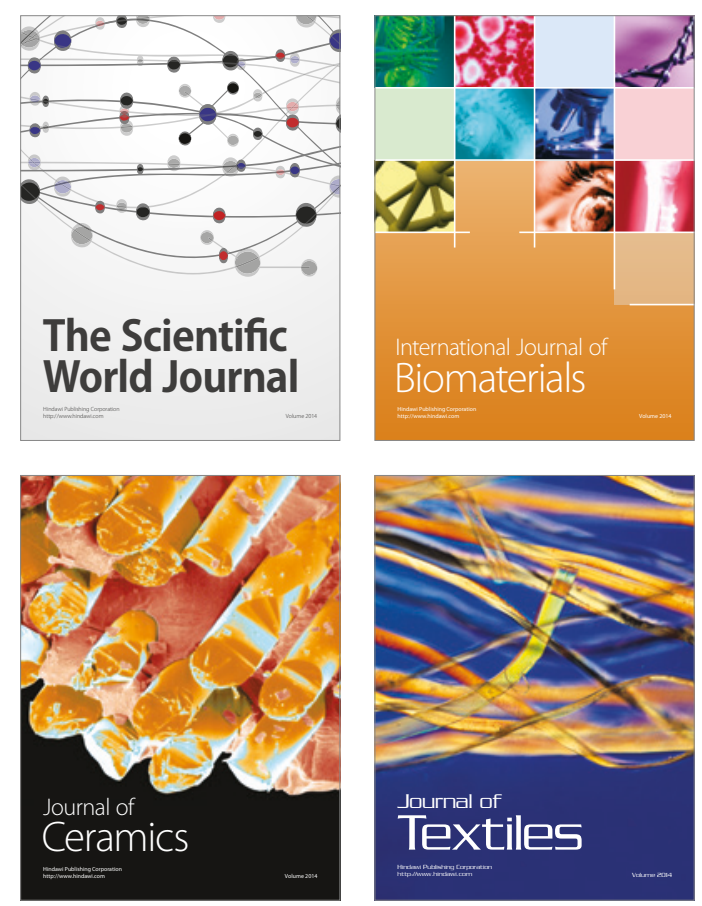